\title{
Magnetotaxis and magnetic particles in bacteria
}

\author{
Richard B. Frankel \\ Department of Physics, California Polytechnic State University, \\ San Luis Obispo, CA 93407, USA \\ and \\ Dennis A. Bazylinski \\ Department of Biology, Marine Science Center, Northeastern University, \\ East Point, Nahant, MA 01908, USA
}

Magnetotactic bacteria contain magnetic particles that constitute a permanent magnetic dipole and cause each cell to orient and migrate along geomagnetic field lines. Recent results relevant to the biomineralization process and to the function of magnetotaxis are discussed.

\section{Introduction}

Magnetotactic behavior in bacteria was discovered about twenty years ago [1]. The discovery was based on the fact that when drops of water and sediment from a number of aquatic habitats are placed on microscope slides in a magnetic field comparable in strength to the geomagnetic field, certain bacteria, of various morphological types [2], but principally cocci, are seen to migrate along magnetic field lines in the field direction, corresponding to Northward migration along geomagnetic field lines [3]. The migration speed of individual bacteria along the magnetic field lines depends on the field strength, but can be $90 \%$ or more of the forward swimming speed (up to 150 microns per second) of the cell. If the direction of the local magnetic field is reversed, the magnetotactic bacteria execute "U-turns" and continue migrating in the same direction relative to the local magnetic field. The migration direction of bacteria in the magnetic field can be reversed by subjecting the cells to a strong (several hundred Gauss) magnetic field pulse oriented opposite to the field in which they are migrating [4]. Magnetotactic bacteria that spontaneously migrate Southward along geomagnetic field lines are found in aquatic sediments and waters from the Southern hemisphere [5]. Review of recent research on magnetotactic bacteria may be found in ref. [6]. 


\section{Magnetic particles in magnetotactic bacteria}

All magnetotactic bacteria contain magnetosomes [7], which are magnetic mineral particles enclosed in membranes. In most cases, the magnetosomes are arranged in a chain or chains [2] apparently fixed within the cell.. In many magnetotactic bacterial types, the magnetosome mineral particles are magnetite, $\mathrm{Fe}_{3} \mathrm{O}_{4}$ [8-12], and are characterized by narrow size distributions, and uniform, species-specific, crystal habits $[13,14]$ (fig. 1). The particle sizes range from approximately 40 to $100 \mathrm{~nm}$, which are within the permanent single-magnetic-domain size range for magnetite.

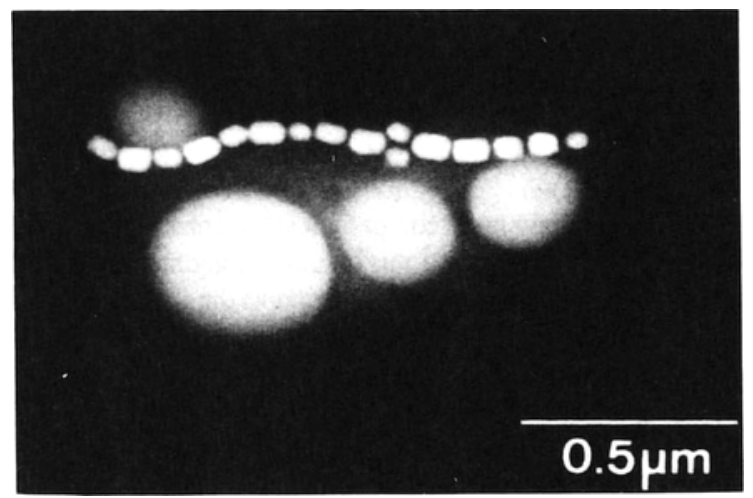

Fig. 1. Dark field transmission electron micrograph of magnetite particles in a magnetotactic bacterium. The spherical, electrondense structures are polyphosphate granules and sulfur granules.

In magnetotactic bacteria from marine, sulfidic environments, the magnetosome particles are the iron-sulfide mineral greigite, $\mathrm{Fe}_{3} \mathrm{~S}_{4}$ [15-18], which is isostrictural with magnetite and is also ferrimagnetically ordered at ambient temperature. The greigite particles are also characterized by narrow size distributions and speciesspecific crystal habits. However, whereas the magnetite particles in a magnetosome chain are usually oriented so that a [111] crystallographic axis of each particle lies along the chain direction, the greigite particles in a magnetosome chain are usually oriented so that a [100] crystallographic axis of each particle is oriented along the chain direction [17].

Whether the mineral particles are magnetite or greigite, the chain of magnetosome particles constitutes a permanent magnetic dipole fixed within the bacterium [3]. The magnetic dipole moment is generally sufficiently large so that it, and consequently the bacterium, is oriented in the geomagnetic field so that $\langle\cos \phi\rangle \approx 0.9$, where $\phi$ is the instantaneous angle between the magnetic dipole moment and the field direction. Magnetotaxis is a passive process in which the orientation of the magnetic dipole 
in the ambient magnetic field as the organism swims causes it to migrate along the magnetic field lines.

Magnetotactic bacteria have two possible magnetic polarities, depending on the orientation of the magnetic dipole within the cell. The polarity can be reversed by a magnetic pulse which is greater than the coercive force of the chain of particles. As noted above, bacteria with reversed polarity migrate along magnetic field lines in the direction opposite to that of bacteria with the original polarity. In natural habitats, the predominant polarity type in the population of a given bacterial species is determined by the sign of the inclination of the geomagnetic field [5].

\section{Magnetotactic bacteria at the OATZ in stratified marine waters}

It has recently been reported that high concentrations of bacteria occur in a horizontal "plate" at the oxic-anoxic transition zone (OATZ) in stratified marine environments [19]. In these environments, downward oxygen diffusion from the surface and upward sulfide diffusion, resulting from bacterial sulfate reduction in

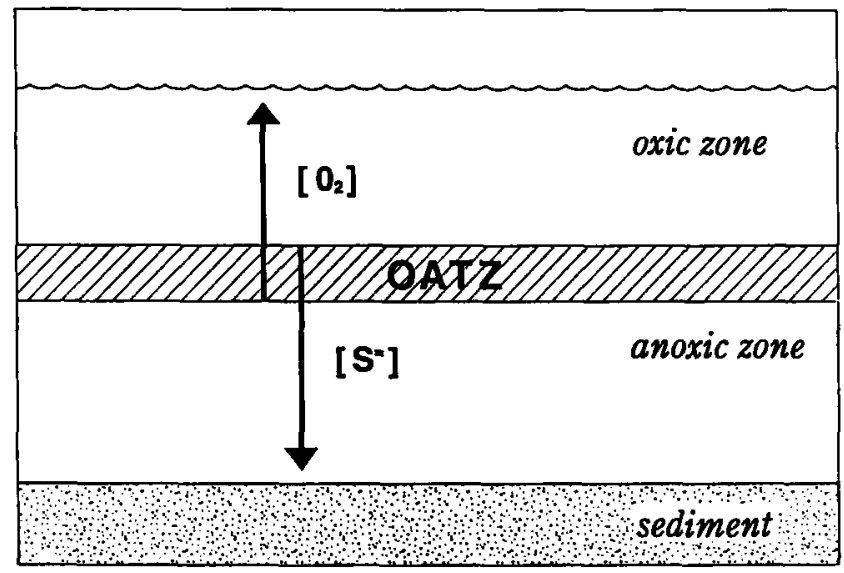

Fig. 2. Illustration (not to scale) of a chemically stratified marine habit with $\mathrm{O}_{2}$ and $\mathrm{S}^{\mathrm{F}}$ concentration gradients (vertical arrows), and an oxic-anoxic transition zone (OATZ). The presence of sulfide is due to bacterial sulfate reduction in the sediment. High concentrations of magnetotactic bacteria are localized in a plate at the OATZ.

the anaerobic sediment, create a double vertical chemical concentration gradient system, with a concomitent redox gradient. In the Pettaquamscutt Estuary in Narragansett Bay, Rhode Island, the OATZ occurs at about $4.5 \mathrm{~m}$ in a $20 \mathrm{~m}$ water column, and the bacterial concentration in the plate is greater than $10^{6}$ cells per $\mathrm{cm}^{3}$. At least seven morphologically-distinct, magnetotactic-bacterial types occur at the OATZ [20-22], some containing $\mathrm{Fe}_{3} \mathrm{O}_{4}$ particles, and some containing $\mathrm{Fe}_{3} \mathrm{~S}_{4}$ particles [22]. The magnetite-containing cells tend to be more abundant in the oxic 
portion of the plate, while the greigite-containing cells tend to be more abundant in the anoxic portion of the plate, in the zone where hydrogen sulfide can be measured.

The close proximity of iron-oxide and iron-sulfide type magnetotactic bacteria at the OATZ raises the question of the relationship of their biomineralization processes. This question has been approached by studying the phylogenetic relations between iron-oxide type and iron-sulfide type magnetotactic bacteria [23]. These were determined from the small subunit ribosomal RNA sequences of two cultured iron-oxide type magnetotactic bacteria and an uncultured iron-sulfide type magnetotactic bacterium, and from previously reported sequences from iron-oxide type magnetotactic bacteria [24-26]. The results showed that all the investigated iron-oxide type bacteria are associated with the alpha subgroups of the Proteobacteria, in the domain Bacteria, whereas the iron-sulfide type bacterium is associated with the sulfate-reducing bacteria within the delta subdivision of the Proteobacteria (fig. 3 ). Thus, the

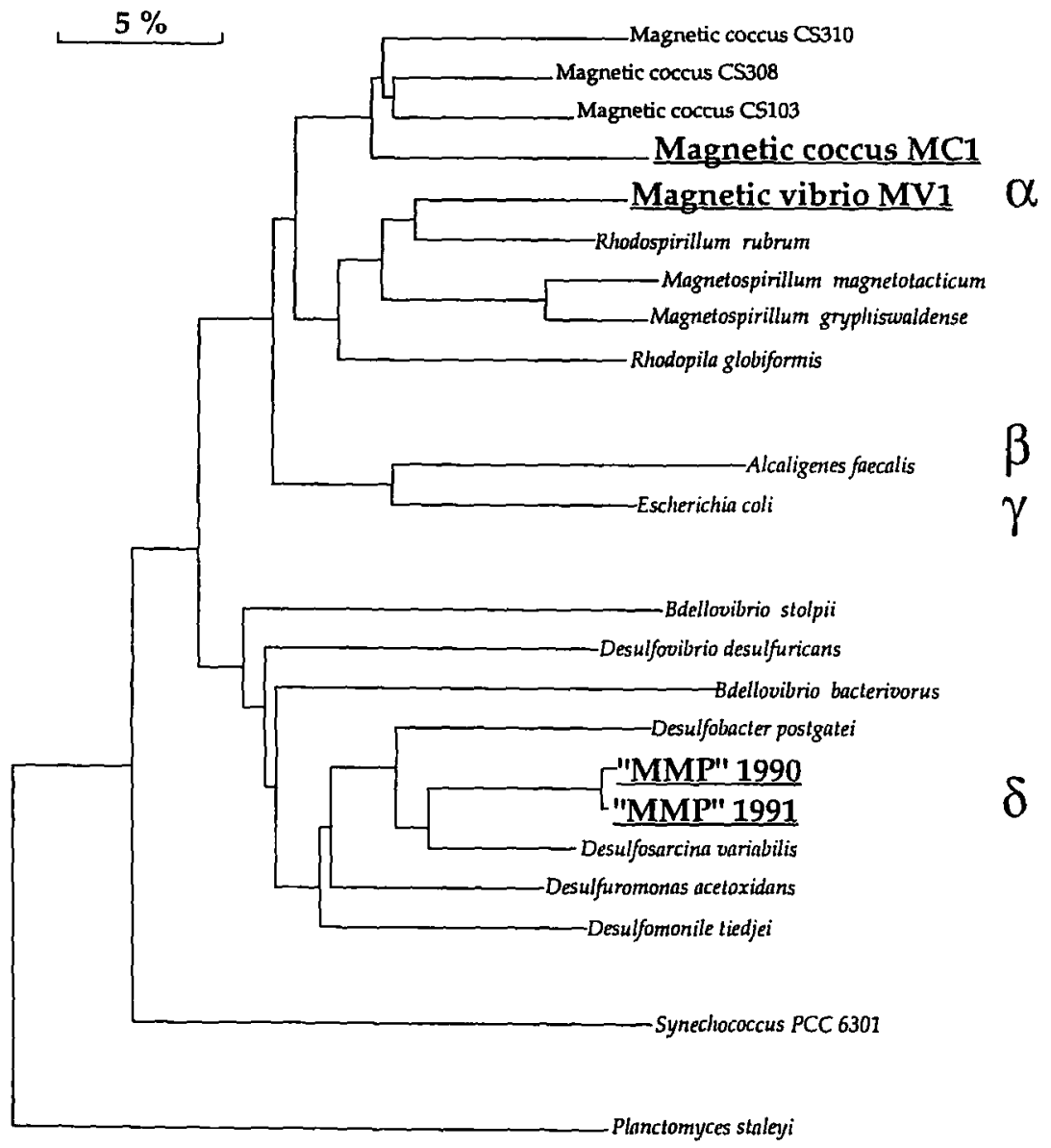

Fig. 3. Phylogenetic relations of iron-oxide type and iron-sulfide type magnetotactic bacteria (see ref. [23]). 
magnetosome mineral could be correlated with phylogenetic affiliation. Because the subdivisions of the Proteobacteria are considered to be distinct, coherent lineages that diverged at an unknown time in the past [27], it is then likely that magnetotaxis based on iron oxides and on iron sulfides has separate evolutionary origins and that the biochemical basis for iron-oxide and iron-sulfide mineralization is fundamentally different [23].

\section{Controlled biomineralization in magnetotactic bacteria}

Although most of the magnetotactic bacteria in the Pettaquamscutt Estuary occur close to the OATZ, large rod-shaped bacteria (approximately $3.1 \mu \mathrm{m}$ by $1.3 \mu \mathrm{m}$ ) are found at the OATZ and also deeper in the water column in the presence of higher hydrogen sulfide and probably no free oxygen [22]. Cells of this organism typically contained two chains of magnetosomes with two morphologically distinct mineral particles, rectangular prisms and elongated tooth-shaped particles (fig. 4). Elemental mapping and electron diffraction was used to show that the particles were greigite and magnetite, respectively [22].

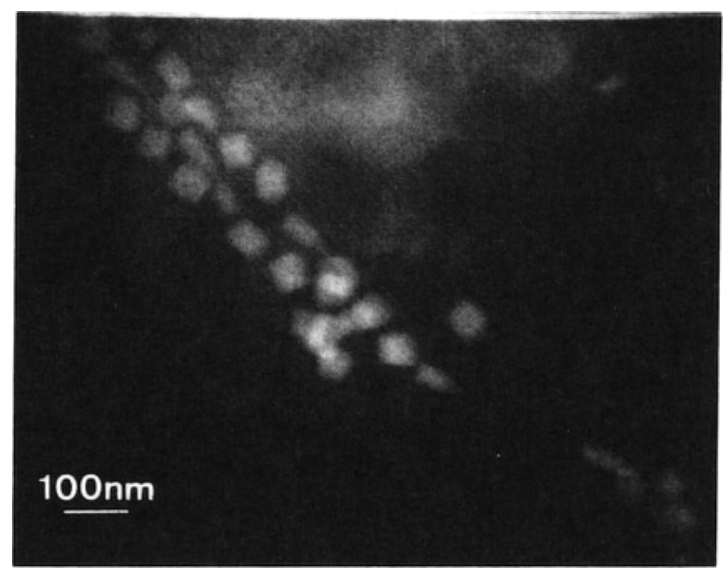

Fig. 4. Transmission electron micrograph of rectangular prismatic greigite particles and tooth-shaped magnetite particles in one and the same magnetotactic bacterium (from ref. [22]).

Thus, whereas all other known magnetotactic bacteria contain either greigite or magnetite particles, this organism is unique in mineralizing both minerals in the same chain or chains of magnetosomes. Both minerals had specific sizes and morphologies, and had their long axes oriented parallel to the chain direction. However, the two minerals had different crystal habits and crystallographic orientation with respect to the chain direction, consistent with different biomineralization processes for the two minerals. 
These results suggest that the processes of (1) particle mineralization, and (2) chain assembly are separately controlled in magnetotactic bacteria. Whereas the particle mineralization processes for the iron-oxide type magnetotactic bateria and the iron-sulfide type magnetotactic bacteria are different, the processes associated with chain assembly and ultrastructure are likely to be common to both groups.

\section{The function of magnetotaxis}

Among the magnetotactic bacteria collected at the OATZ in the Pettaquamscutt Estuary were cocci $[13,23]$ that exhibited the same persistent North-seeking motility when examined in drops of water on microscope slides as that exhibited by sedimentdwelling cocci (see the introduction). This raises the question of how these bacteria maintain their position in the OATZ, instead of migrating down the inclined magnetic field lines to the anaerobic sediments at the bottom of the water column. We propose these these bacteria can actually swim in both directions along the geomagnetic field lines without turning around, with the direction of motility determined by local environmental conditions such as oxygen concentration, sulfide concentration, and/or redox potential. According to this proposal, the bacteria are North-seeking, i.e. they migrate in the field direction, hence downward along the inclined geomagnetic field lines, when the oxygen concentration or redox potential is higher than optimal and/or the sulfide concentration is lower than optimal. On the other hand, when the oxygen concentration, or the redox potential, is too low, or the sulfide concentration too high, they migrate opposite to the field direction, upward along the inclined geomagnetic field lines (fig. 5). When they are examined in drops on microscope slides, diffusion of oxygen into the drop makes the oxygen concentration and the redox potential higher than optimal and the sulfide concentration lower than optimal, causing the cells to migrate persistently along the geomagnetic field lines in the field direction, i.e. exhibit North-seeking magnetotaxis.

Similar bacteria in the Southern hemisphere would also maintain position in vertical concentration gradients by migrating downward along the inclined geomagnetic field lines when the oxygen concentration or the redox potential is too high, or the sulfide concentration too low, and migrating upward when the oxygen concentration and the redox potential are too low and the sulfide concentration too high. Because the geomagnetic field lines are now inclined upward, downward migration would now require migration opporite to the field direction. This would occur for bacteria with a polarity opposite to that of similar bacteria in the Northern hemisphere [3]. When examined in water drops with relatively high concentrations of oxygen due to diffusion, these Southern hemisphere bacteria would migrate persistently opposite to the field direction, i.e. exhibit South-seeking magnetotaxis.

In both hemispheres, magnetotaxis is especially advantageous to organisms in vertical concentration gradients because it presumably increases the efficiency 


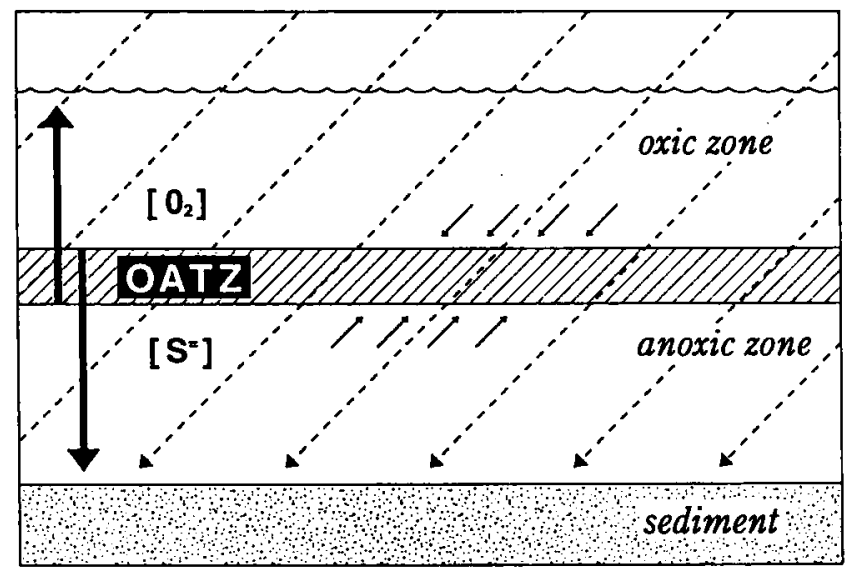

Fig. 5. Schematic representation of how magnetactic bacteria could maintain their position at the OATZ in a vertical chemical concentration gradient system (vertical arrows) When cells are above the OATZ, they would swim downward (small arrows above OATZ) along the inclined geomagnetic field lines (dashed lines). When they are below the OATZ, they would reverse direction, possibly by reversing the direction of flagellar rotation, and swim upward (small arrows below OATZ) along the inclined geomagnetic field lines. The direction of flagellar rotation could be coupled to a sensory system that responds to chemical concentration or redox potential.

of maintaining an optimal position relative to the gradient by reducing a threedimensional search problem to a one-dimensional problem. Thus, magnetotaxis is partially passive in that it obliges the cell to migrate along geomagnetic field lines, and partially active, if there is another sensory input that controls the direction of migration, i.e. parallel or antiparallel to the field direction.

We may also speculate how cells determine the direction of migration. Bacteria swim by rotating their helical flagella $[28,29]$. Clockwise rotation would result in the cell moving in one direction, while counter-clockwise rotation would result in the cell moving in the opposite direction. If a magnetotactic bacterium has a sensory input, responsive to local chemical conditions, that is coupled to the sense of flagellar rotation, the cell could migrate along the ambient magnetic field lines in a direction determined both by the chemical or redox levels and by the orientation of the cellular magnetic dipole with respect to the flagella.

In many freshwater and in shallow marine environments, the OATZ is located at the sediment-water interface, or even below the surface of the sediments. In these situations, the bacteria could conceivably stop swimming or attach themselves to sediment particles in the OATZ, and use their magnetotactic response to return to the OATZ when they are dislodged into the relatively more oxidizing water above the sediments. 


\section{Acknowledgements}

We are grateful to Professor $\mathrm{H}$. de Waard for a critical reading of the manuscript. This work was supported by U.S. Office of Naval Research Contract No. N0001491-J-1290. DAB was also supported by U.S. National Science Foundation Grant No. MCB-9117694.

\section{References}

[1] R.P. Blakemore, Science 190(1975)377.

[2] R.P. Blakemore, N.A. Blakemore, D.A. Bazylinski and T.T. Moench, in: Bergey's Manual of Systematic Bacteriology, Vol. 3, eds. M.P. Bryant, N. Pfenning and J.T. Staley (Williams and Wilkins, Baltimore, 1989) p. 1882.

[3] R.B. Frankel, Ann. Rev. Biophys. Bioeng. 13(1984)85.

[4] A.J. Kalmijn and R.P. Blakemore, in: Animal Migration and Homing, eds. K. Schmidt-Koenig and W.T. Keeton (Springer, New York, 1978) p. 344.

[5] R.P. Blakemore, R.B. Frankel and A.J. Kalmijn, Nature 286(1980)384.

[6] R.B. Frankel and R.P. Blakemore (eds.), Iron Biominerals (Plenum Press, New York, 1990).

[7] D.L. Balkwill, D. Maratea and R.P. Blakemore, J. Bacteriol. 141(1980)1399.

[8] R.B. Frankel, R.P. Blakemore and R.S. Wolfe, Science 203(1979)1355.

[9] K.M. Towe and T.T. Moench, Earth Planet. Sci. Lett. 52(1981)213.

[10] T. Matsuda, J. Endo, N. Osakabe, A. Tonomura and T. Arii, Nature 343(1983)258.

[11] S. Mann, N.H.C. Sparks and R.P. Blakemore, Proc. Roy. Soc. London B231(1987)469.

[12] D.A. Bazylinski, R.B. Frankel and H.W. Jannasch, Nature 334(1988)518.

[13] F.C. Meldrum, B.R. Heywood, S. Mann, R.B. Frankel and D.A. Bazylinski, Proc. Roy. Soc. London B251(1993)231; 237

[14] S. Mann and R.B. Frankel, in: Biomineralization: Chemical and Biological Perspectives, eds. S. Mann, J. Webb and R.J.P. Williams (Springer, Berlin, 1990) pp. 389-426.

[15] S. Mann, N.H.C. Sparks, R.B. Frankel, D.A. Bazylinski and H.W. Jannasch, Nature 343(1990)258.

[16] B.R. Heywood, D.A. Bazylinski, A.J. Garratt-Reed, S. Mann and R.B. Frankel, Naturwissensch. 77(1991)536.

[17] B.R. Heywood, S. Mann and R.B. Frankel, in: Materials Synthesis Based on Biological Processes, eds. M. Alpert et al. (Mater. Res. Soc., Pittsburgh, PA, 1990) p. 93.

[18] M. Farina, D.M.S. Esquivel and H.G.P. Lins de Barros, Nature 334(1990)256.

[19] P.L. Donaghay, H.M. Rines and J. McN. Sieburth, Arch. Hydrobiol. Beih. Ergbn. Limnol. 36(1992)96.

[20] J.F. Stolz, in: Biomineralization Processes of Iron and Manganese - Modern and Ancient Environments, eds. H.W.C. Skinner and R.W. Fitzpatrick (Catena, Cremlingen-Destedt, 1992) p. 133.

[21] J.M. Sieburth, P.L. Donaghay, P.W. Johnson and J.F. Stolz, submitted for publication.

[22] D.A. Bazylinski, B.R. Heywood, S. Mann and R.B. Frankel, Nature 366(1993)218.

[23] E.F. DeLong, R.B. Frankel and D.A. Bazylinski, Science 259(1993)803.

[24] P.A. Eden, T.M. Schmidt, R.P. Blakemore and N.R. Pace, Int. J. Syst. Bacteriol. 41(1991)324.

[25] K.H. Schleifer et al., Syst. Appl. Microbiol. 14(1991)379.

[26] S. Spring, R. Amann, W. Ludwig, K.H. Schleifer and N. Petersen, Syst. Appl. Microbiol. 15(1992)116.

[27] C.R. Woese, Microbiol. Rev. 51(1987)51.

[28] H.C. Berg, Ann. Rev. Biophys. Bioeng. 4(1975)119.

[29] R.M: MacNab and S.I. Aizawa, Ann. Rev. Biophys. Bioeng. 13(1984)51. 